\title{
Staphylococcal scalded skin syndrome: A case report
}

\section{Leila Bouhajja', Anissa Zaouak', Ehsen Ben Brahim², Selima Ben Jannet', Houda Hammami ${ }^{1}$, Samy Fenniche ${ }^{1}$}

${ }^{1}$ Department of Dermatology, Habib Thameur Hospital, Tunis, Tunisia, ${ }^{2}$ Department of Pathology, Habib Thameur Hospital, Tunis, Tunisia

Corresponding author: Dr. Leila Bouhajja, E-mail: bouhaja.leila@gmail.com

\begin{abstract}
Staphylococcal Scalded Skin Syndrome (SSSS) is an extensive and exfoliative skin disease which is caused by staphylococcal epidermolytic toxin produced by Staphylococcus aureus. A 20-month-old female infant presented with a desquamating rash with flaccid blisters and fever. Skin biopsy revealed superficial intraepidermal cleavage under the stratum corneum. The diagnosis of scalded skin syndrome was assessed and the patient was treated with antistaphylococcal antibiotics with a complete recovery of her skin lesions. SSSS is a pediatric emergency, representing a fatal condition in neonates and a challenge in diagnostic and treatment. Therefore, early diagnosis, prompt treatment, and following strict hygiene measures are imperative and can prevent mortality.
\end{abstract}

Key words: Scalded skin syndrome; Desquamation; Staphylococcus aureus; Exfoliative toxins

\section{INTRODUCTION}

Staphylococcal Scalded Skin Syndrome (SSSS) is an extensive and exfoliative skin disease which is caused by Staphylococcal epidermolytic toxin produced by Staphylococcus aureus. It occurs in newborns, children less than 5 years of life, and adults with various comorbidities $[1,2]$. We report a new case of SSSS in a 20- month-old female infant.

\section{CASE REPORT}

A 20-month-old female infant presented with fever and a generalized rash since 4 days. There was no family history of similar skin lesions. On physical examination, skin was tender with diffuse erythema and she had superficial erosions and peeling on her trunk (Fig. 1). The superficial erosions started on the axillary region extending to the trunk and limbs with sparing of the mucosa. There were flaccid blisters that ruptured leading to superficial erosions with exfoliation. Nikolsky's sign was positive on the trunk. Other body system examinations were normal.
A skin biopsy was performed on her trunk showing superficial intraepidermal split into the granular layer associated with little inflammatory infiltrate in the superficial dermal layer (Fig. 2). Hence, the diagnosis of SSSS was assessed and the patient was treated with antistaphylococcal antibiotics, administred intravenously $100 \mathrm{mg} / \mathrm{kg} / \mathrm{day}$ for 10 days with a complete recovery of her skin rash.

Prior to the study, patient gave written consent to the examination and biopsy after having been informed about the procedure.

\section{DISCUSSION}

Staphylococcal scalded skin syndrome (SSSS), also known as Ritter's disease, is a rare skin infection seen in neonatal period and early childhood as in our patient. It is a potentially life-threatening skin disorder caused by certain strains of staphylococcus aureus that release serine protease exfoliate toxins, exotoxin A (ETA), the most produced one, and the exotoxin $\mathrm{B}(\mathrm{ETB})$. These two exfoliative toxins have an affinity to the

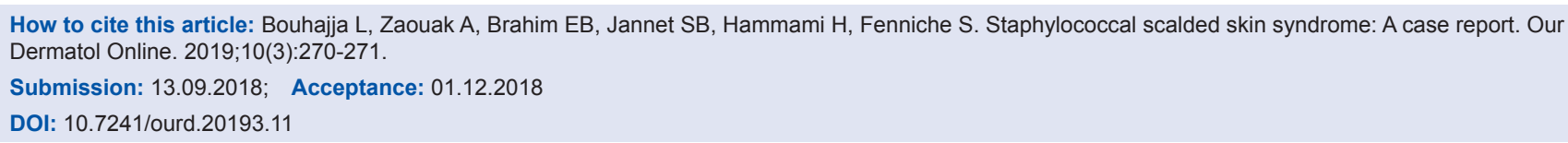




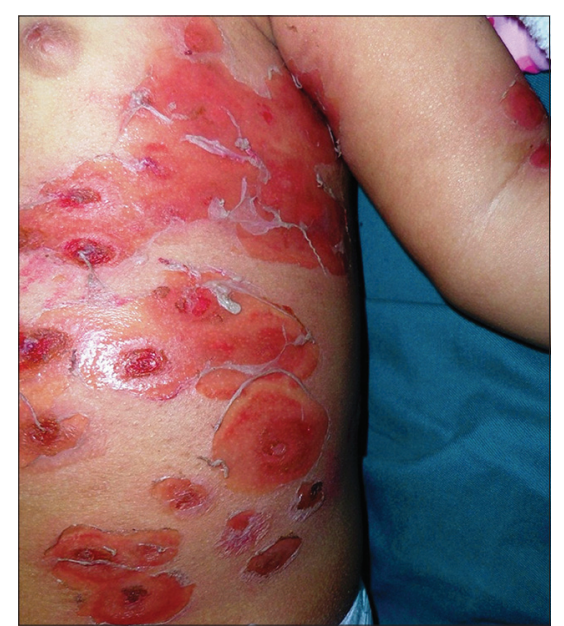

Figure 1: Superficial epidermal peeling on the trunk.

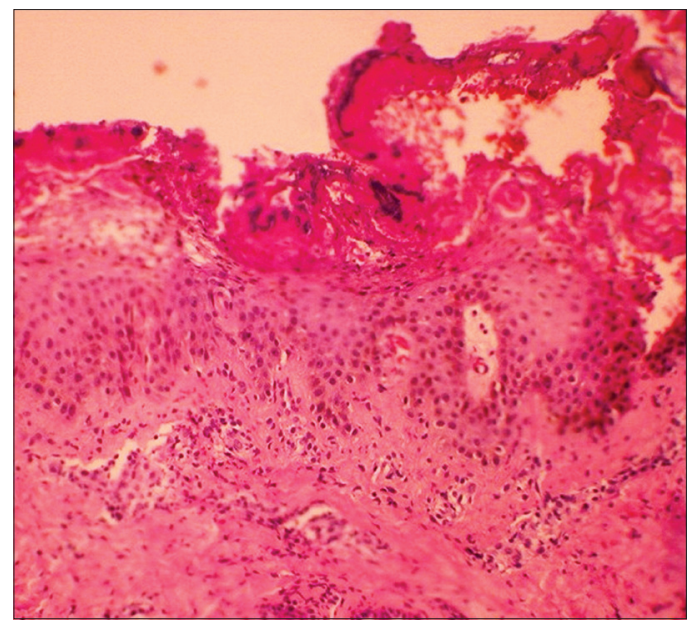

Figure 2: Superficial intraepidermal cleavage under the stratum corneum (HEx100).

glycoprotein, desmoglein-1, present on desmosomes located in the zona granulosa layer of the skin, so they cleave desmosomal cadherins, destroy adhesion between the keratinocytes, leading to epidermolysis and bulla formation $[1,2]$. Moreover, the lack of protective antibodies to exfoliative toxins and immature renal function, explain the higher risk of SSSS in neonates and children. The diagnosis of SSSS is mainly clinical. It usually presents as generalized erythematous extensive skin lesions associated with the formation of large fragile roofed superficial blisters leading to extended areas of eroded skin, bullae, and desquamation with a scalded appearance especially in friction zones, periorificial crusting, and positive Nikolsky sign [3]. This disease may resemble toxic epidermal necrosis which is a life threatening skin disorder. However, in SSSS there is absence of mucosal involvement as well as superficial epidermal peeling with absence of necrotic keratinocytes characteristic of toxic epidermal necrosis. It could also resemble pemphigus foliaceous and bullous impetigo (BI) but in BI, the skin lesions are smaller and there is pronounced inflammatory cell infiltrate consisting mostly in neutrophils and the skin $[1,3,4]$. A prompt administration of intravenous antibiotics mainly antistaphylococcal penicillins generally allows a favorable outcome [5]. Recovery is achieved after 6 to 12 days without scarring. Prognosis is mostly favorable if treatment is begun promptly, and the mortality rate is $4 \%$, due to serious complications like pneumonia, septic arthritis, hypothermia, dehydration, and secondary infections [4].

\section{CONCLUSION}

SSSS is a rare cause of skin infection caused by staphylococcus aureus. It is a pediatric emergency, representing a fatal condition in neonate and a challenge in diagnostic and treatment. Therefore, early diagnosis, prompt treatment, and following strict hygiene measures are imperative and can prevent the mortality.

\section{Consent}

The examination of the patient was conducted according to the Declaration of Helsinki principles. Written informed consent was obtained from the child's parents for publication of this article.

\section{REFERENCES}

1. Handler MZ, Schwartz RA. Staphylococcal scalded skin syndrome: diagnosis and management in children and adults. J Eur Acad Dermatol Venereol. 2014;28:1418-23.

2. Meshram GG, Kaur N, Hura KS. Staphylococcal scalded skin syndrome: A pediatric dermatology case report. AGE Open Med Case Rep. 2018;6:2050313X17750890.

3. Kouakou K, Dainguy ME, Kassi K. Staphylococcal Scalded Skin Syndrome in Neonate. Case Rep Dermatol Med. 2015;2015:901968.

4. Oliveira AR, Aires S, Faria C, Santos E. Staphylococcal scalded skin syndrome.BMJ Case Rep. 2013;2013:pii: bcr2013009478.

5. Jindal S, Bhobhe M, Jerajani H. An infant with skin rash.Indian Pediatr. 2012;49:853-4.

Copyright by Leila Bouhajja, et al. This is an open-access article distributed under the terms of the Creative Commons Attribution License, which permits unrestricted use, distribution, and reproduction in any medium, provided the original author and source are credited.

Source of Support: Nil, Conflict of Interest: None declared. 